\title{
BMJ Open Supporting population mental health and wellness during the COVID-19 pandemic in Canada: protocol for a sequential mixed-method study
}

\author{
Tracie Risling, ${ }^{1}$ Courtney Carlberg, ${ }^{1}$ Iman Kassam, ${ }^{2}$ Tyler Moss, ${ }^{1}$ Patricia Janssen, ${ }^{3}$ \\ Steve Iduye, ${ }^{1}$ Gillian Strudwick (1) ${ }^{2,4}$
}

To cite: Risling T, Carlberg C, Kassam I, et al. Supporting population mental health and wellness during the COVID-19 pandemic in Canada: protocol for a sequential mixedmethod study. BMJ Open 2021;11:e052259. doi:10.1136/ bmjopen-2021-052259

- Prepublication history and additional supplemental material for this paper are available online. To view these files, please visit the journal online (http://dx.doi.org/10.1136/ bmjopen-2021-052259).

Received 14 April 2021 Accepted 29 October 2021

Check for updates

(C) Author(s) (or their employer(s)) 2021. Re-use permitted under CC BY-NC. No commercial re-use. See rights and permissions. Published by BMJ.

${ }^{1}$ College of Nursing, University of Saskatchewan, Saskatoon,

Saskatchewan, Canada

${ }^{2}$ Campbell Family Mental Health Research Institute, Centre for Addiction and Mental Health, Toronto, Ontario, Canada

${ }^{3}$ School of Population and Public Health, University of British Columbia, Vancouver, British Columbia, Canada

${ }^{4}$ Institute of Health Policy, Management and Evaluation, University of Toronto, Toronto, Ontario, Canada

Correspondence to

Dr Gillian Strudwick; gillian.strudwick@camh.ca

\section{ABSTRACT}

Introduction The global COVID-19 pandemic has reported to have a negative impact on the mental health and well-being of individuals around the world. Mental health system infrastructure, primarily developed to support individuals through in-person care, struggled to meet rising demand for services even prior to COVID-19. With public health guidelines requiring the use of physical distancing during the pandemic, digital mental health supports may be one way to address the needs of the population. Despite this, barriers exist in promoting and supporting access to existing and emerging digital resources. Text messaging may address some of these barriers, extending the potential reach of these digital interventions across divides that may separate some vulnerable or disadvantaged groups from essential mental health supports. Building on an existing knowledge synthesis project identifying key digital resources for improved mental health, this research will establish lowtech connections to assess need and better match access to services for those who need it most. The aim of this study is to codesign a customised two-way texting service to explore need and better align access to mental health supports for Canadians located in Saskatchewan during the COVID-19 pandemic.

Methods and analysis This study will be completed in Saskatchewan, Canada. For this project, the REAIM (reach, effectiveness, adoption, implementation, maintenance) framework will be used to support three phases of a sequential mixed-method study. An advisory committee of Saskatchewan residents will guide this work with the study team. A 10-week service will be launched to connect individuals with appropriately suited digital mental health interventions through the use of text messaging. In phase 1 , implementation and prototyping will be conducted with collaborative codesign for key elements related to features of an enrolment survey and initial messaging content. Phase 2 will focus on advancing the effectiveness of the service using quantitative user data. In phase 3 , an embedding approach will be used to integrate both qualitative and quantitative data collected to understand the overall acceptability, satisfaction and perceived benefit of the text messaging service. Thematic analysis and descriptive statistics will be used as analytic methods.
Strengths and limitations of this study

- A strength of the study is the engagement of an advisory committee consisting of residents of Saskatchewan in the design and decisions related to the study use.

- Another strength of this research is the use of three 10-week iterative testing cycles to refine the service that participants take part in using the RE-AIM framework.

- The quantitative data collected in this study will supplement the qualitative findings, increasing the breadth and depth of the results.

- A limitation of this study is the limited availability of digital mental health interventions to individuals with limited access to technology, poor connectivity or a non-smartphone.

Ethics and dissemination This study has received approval from the Research Ethics Board at the University of Saskatchewan. A knowledge dissemination plan has been developed that includes traditional academic approaches such as conference presentations, and academic publications, as well as mainstream approaches such as social media, radio and dissemination through the advisory committee.

\section{INTRODUCTION}

As a result of the COVID-19 pandemic, preventative public health measures across Canada have been imposed to curb the rapid spread and detrimental physical health impacts of the COVID-19 virus. ${ }^{12}$ Preventative measures, inclusive of physical and social distancing, have been shown to support infection control; however, the effects of physical distancing and social isolation, coupled with the uncertainty and fear created by the swift onset of COVID-19, has been described by some as an 'echo pandemic' of declining distress and mental health among the Canadian population. ${ }^{34}$ A Canadian survey conducted in May 2020 noted $38 \%$ of individuals attributed a 
decline in their mental health to COVID-19 and $46 \%$ felt anxious and worried. ${ }^{5}$ In September 2020, researchers repeated this national survey and found $40 \%$ of Canadians reported their mental health had declined due to COVID-19 and $48 \%$ of individuals expressed feelings of anxiety or worry. ${ }^{56}$ The negative impact of the COVID-19 pandemic on the distress and mental health of Canadians warrants concern as self-reported population mental health continues to decline. ${ }^{47-9}$ Given the potential strain that the COVID-19 pandemic could create on an already overburdened and overwhelmed mental healthcare system, a long-lasting and sustainable response is needed to support mental healthcare delivery and proactively assist individuals in maintaining mental wellness. ${ }^{14}$ One solution that may support the provision and scaling of mental healthcare is digital health. Current digital health innovations include, but are not limited to, mobile apps, virtual care, chatbots and text messaging platforms and can be used to support improved scalability, flexibility and accessibility of mental healthcare service delivery. ${ }^{2}{ }^{10}$ In particular, text messaging services have the potential to support the mental health of the general population in addition to underserved and marginalised populations. $^{210}$

Mobile phone use continues to expand in Canada. The Canadian Wireless Telecommunications Association (CWTA) reported 34.1 million wireless subscriptions in the country noting more mobile phones $(91.0 \%)$ than landlines $(37.8 \%) .{ }^{11}$ Researchers have capitalised on the ubiquitous spread of mobile devices globally and demonstrated the value of texting programmes in influencing positive health behaviours, ${ }^{12-14}$ especially when messaging can be tailored to individuals or population groups. ${ }^{15} 16$ There are multiple advantages to pursuing a texting-based approach to citizen engagement. First is the immediacy of the connection. Research has demonstrated that ' $90 \%$ of all mobile text messages are read within three min of being received'. ${ }^{17}$ Second, as previously noted, the use of SMS texting is a means to address potential information or digital divide among priority populations facing socioeconomic or other challenges. ${ }^{12} 13$ This includes reliable internet connectivity, which is an ongoing issue in rural and remote communities across Canada. There is an existing study supporting two-way messaging as an engaging and immediate way to employ communitybased surveying ${ }^{18}$ and others that note this as a means to deliver essential public information. ${ }^{17}{ }^{19}$ In the text-based initiative SmartMom Canada, the critical importance of patient partnership in the design and delivery of texting programmes, especially those planned at a population level, was noted. ${ }^{16}$ This approach may also be useful to support those individuals who may 'ration' digital connectivity due to limited data or internet access but often have some point(s) of connection. ${ }^{20}$

The WHO has stated COVID-19 'is the first pandemic in human history where technology and social media are being used on a massive scale to keep people safe, productive and connected'. ${ }^{21}$ With 4 and 5 billion global users of internet and mobile phones, respectively, the reach of these technologies has never been more ubiquitous. ${ }^{22}$ The WHO has also noted the impact of COVID-19 on global mental health identifying the tremendous stressors associated with this world event. ${ }^{23}$ With over 34 million active wireless subscribers in Canada, ${ }^{11}$ SMS text messaging is an established conduit through which to explore need and facilitate access to mental health services. Incorporating two-way communication via text in health programming has revealed considerable value for patients and/or target populations in a systematic review ${ }^{17}$ and research. ${ }^{1224-26}$ Establishing an interactive COVID-19 texting service will extend the reach of existing digital mental health resources as researchers explore solutions with those who may have limited connectivity and work to address digital barriers to identified supports.

\section{METHODS AND ANALYSIS}

\section{Study purpose and objectives}

The aim of this study is to codesign a customised two-way texting service to explore need and better align access to mental health supports for the general Canadian population living in Saskatchewan during the COVID-19 pandemic. Working in collaboration with Saskatchewan residents and building on a currently funded study, timely, relevant and population-specific mental health information and resources can be shared. In addition, the two-way texting function will allow immediate opportunities for community engagement with polling questions employed to identify unmet needs and challenges in accessing digital mental health resources. This low-tech approach can increase connections to populations that may not be aware of existing digital supports. Issues of digital exclusion often include limited data plans and reliable internet service, as well as underlying challenges related to digital health literacy. ${ }^{2027}$ In order to identify solutions to these barriers, Brewer $e t a l^{28}$ called on digital health researchers to 'embrace implementation science and community engagement in our collective quest to eliminate health disparities' ${ }^{28}$ In answering that call, this project will employ the RE-AIM (reach, effectiveness, adoption, implementation, maintenance) framework, ${ }^{29}$ a well-established implementation tool for planning and evaluating public health initiatives, to monitor the reach, effectiveness, adoption, implementation and maintenance of the planned texting service through three phases of a mixed methods design. ${ }^{29}$

The following research objectives will guide these efforts:

1. Codesign a texting service to assess and support improved reach of mental health services in collaboration with a resident advisory committee in a Canadian jurisdiction.

2. Optimise the effectiveness of the texting service through collaborative iterative testing cycles.

3. Evaluate the adoption of the texting service with a focus on acceptability, satisfaction and benefit. 


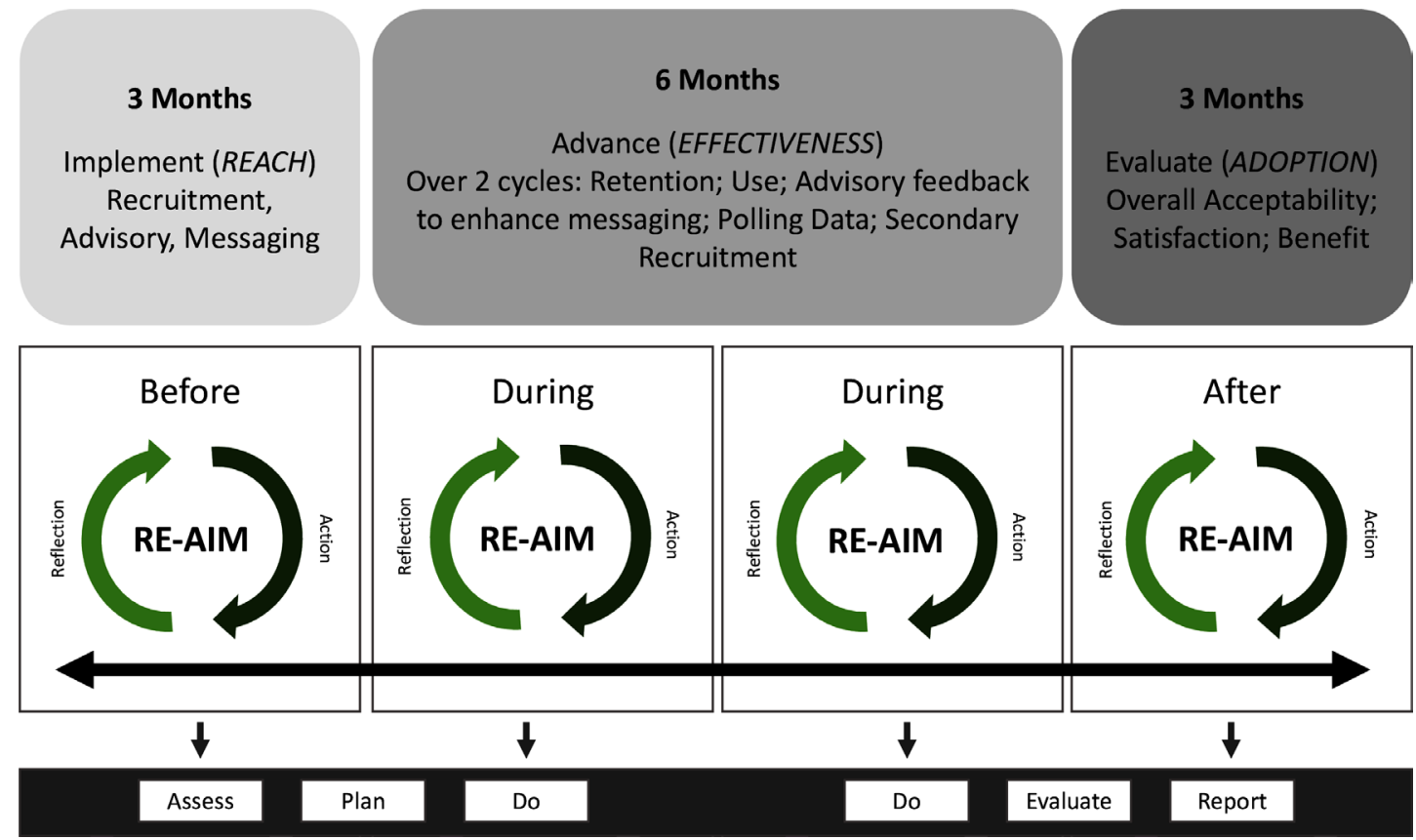

Figure 1 Adapted from Harden et al ${ }^{31}$ (2018) RE-AIM method map. RE-AIM, reach, effectiveness, adoption, implementation, maintenance.

Owing to the unpredictability of the pandemic trajectory, this project will focus on the first three RE-AIM framework elements with implementation and maintenance analysis used to advance future scalability.

\section{Study design}

For this project, the RE-AIM framework will be used to support three phases of a mixed-methods study. This framework has previously been used to guide and evaluate a SMS project. ${ }^{30}$ The project plan (figure 1) is built on a modified figure from Harden et al detailing an iterative and temporal application of the RE-AIM approach. ${ }^{31}$ In this case, instead of employing a single 'during' cycle, this adapted figure denotes the two rounds of collaborative testing for this project. The three study objectives each anchor a project phase coupled with a primary RE-AIM focus. The project commenced in March 2021 and is expected to continue until December 2021. In phase 1, implementation and prototyping will be launched with collaborative codesign on key elements related to features of a web-based enrolment survey and initial messaging content. Further details of the web-based enrolment survey can be found in online supplemental appendix A. Planned message frequency will be approximately two times per week, with an additional 'polling' option that will see the weekly delivery of short research surveys. Qualitative data from focus groups and early-adopter interviews will be a driver in this phase as the team seeks to maximise the reach of the service. Quantitative demographic enrolment and usage data collection will also begin. Phase 2 will focus on advancing the effectiveness of the texting service relying more heavily on quantitative user data provided from the web-based portal. Response, retention and survey completion rates will address reach, while enrolment and survey data are used to tailor messaging to advance the effectiveness of the intervention in increasing service awareness and access. The 'polling' questions will be used as an ongoing assessment of needs and access challenges. While quantitative data is the primary focus of this phase, qualitative interviewing will continue to provide essential context to usage statistics along with monthly virtual meetings with our Saskatchewan resident advisor committee. During the evaluation process in phase 3 , both qualitative and quantitative data will be used to address overall acceptability, satisfaction and perceived benefit of the text messaging service. Final quantitative participant and usage data analysis will cue further explanatory qualitative inquiry. This will include stakeholder interviews with our collaborators and other healthcare practitioners to ascertain intervention impact, user interviews and a final advisory debriefing.

\section{Study population, recruitment and sampling}

While the goal of this work is to achieve a national SMS service, this study will focus on developing and examining feasibility in one Canadian province, Saskatchewan. The province is an ideal incubator for this work with a manageable population size that includes diverse representation of the user bases (eg, urban, rural, remote, populations with varied SES and digital connectivity). Given this, the target population includes the general population of Saskatchewan who meet the following inclusion criteria: 16 years of age or older, with ready access to a personal or family-shared mobile phone and an ability to understand English language recruitment and messaging. A representative sample will be sought for using targeted recruitment through community newsletters, social media, radio public service announcements and snowball 
sampling to engage low-tech users. Participant enrolment demographics will be monitored by the study team. Recruitment targets and promotion will then be revised based on learning and suggestions of the advisory group. This approach is meant to provide opportunity and flexibility for a wide range of analysis and exploration, as needed. For example, purposive sampling supports the recruitment of participants with in-depth knowledge of the phenomenon under study ${ }^{32}$ and will be essential to fulfil the evaluative qualitative needs of this study. Braun and Clarke ${ }^{33}$ recommend 6-10 participant interviews for the successful completion of a focused thematic analysis and this will guide the research team members in forming participant groupings to achieve qualitative priorities.

\section{Text-based service}

The two-way texting service, entitled 'SaskWell', will provide users with mental health and wellness content for the duration of ten weeks. Users will be able enrol in SaskWell through four different means, scanning a $Q R$ code that will populate the enrolment survey link, texting 'JOIN' to a national short code (759355), directly clicking on enrolment survey link or calling a toll-free number (1-855-237-5934), which was established for the purpose of this project. Interested users must fill-out an enrolment survey in order to participate in the SaskWell service. The survey consists of three sections. The first section asks participants a series of demographic questions (eg, race/ ethnicity, age, gender and place of residence). The second section pertains to the user's technology and internet access, whereby users are asked whether they have access to a mobile device and whether they have stable and reliable internet access. Finally, the third section aims to understand the user's mental health status. A validated 14-item questionnaire, developed by Corey Keyes et $a l,{ }^{34}$ has been incorporated into the enrolment survey to understand whether a user has flourishing, moderate or languishing mental health. The detailed enrollment survey can be found in online supplemental Appendix A.

Participants will receive a digital mental health tool based on the results of their technology assessment and, if indicated during the enrollment survey, they may receive a digital mental health tool that is specific to their age or race. In the second week of the service, a text message will follow-up with the participant to see if: (1) the participant used their digital mental health tool; (2) if the participant liked the tool they received; and (3) if they would like to receive a new tool. If participants indicate they would like another digital mental health tool, they will be provided with a new tool. In addition to the digital mental health tool, participants will receive weekly wellness resources (eg, psychoeducation resources which include how to practice mindfulness, self-care, guided imagery and meditation, etc) and polling questions (eg, how did you hear about SaskWell?; why did you choose to register for the service?). At the midpoint (week 5) and endpoint (week 10), participants are asked to redo the mental health selfcheck survey to measure the impact of SaskWell. Participation in the service is entirely voluntary and users can opt out of service at any point. As previously noted, the service will provide content to participants for 10 weeks, and three iterations are planned. During each iteration the research team will ask participants during the weekly polling question (week 8) if they are interested in participating in a short interview. A semistructured interview guide (online supplemental Appendix B) has been prepared to support the facilitation of these interviews. An enrolment flow diagram is shown in figure 2.

\section{Digital mental health interventions included in the service}

The digital mental health tools included in the SaskWell service were curated from a knowledge synthesis study completed by researchers at the Centre for Addiction and Mental Health in May-November $2020 .{ }^{9}$ In this study, a rapid review of the academic and grey literature was conducted to identify digital health interventions that could be used to support population mental health in Canada during and beyond the COVID-19 pandemic. From the searches, a total of 114 web-based resources (eg, wellness hubs, telemedicine, self-guided courses, discussion forums, phone/texting services, virtual peer support, etc) and 31 mobile apps were identified and compiled into a database using tags to categorise and sort the various interventions. The full list of digital mental health interventions can be found in ref ${ }^{35}$. For the purpose of the SaskWell service, interventions that are free of cost and accessible to residents of Saskatchewan will be included in the first iteration of the service.
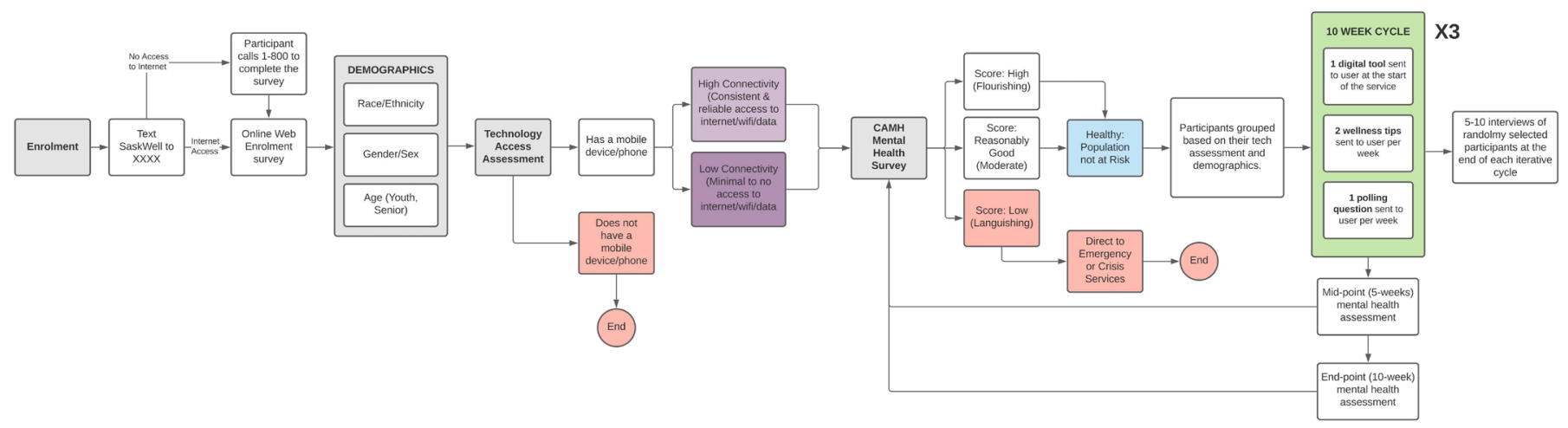

Figure 2 Enrolment flow diagram. 
Further details about the digital mental health interventions included in the SaskWell service can be found by viewing the Airtables (web-based resources: https:// airtable.com/shr4Zh7RDBT4oTsd8 and mobile apps: https://airtable.com/shrPtm50fuJEP93vq).

\section{Data collection and analysis}

Data collection will be facilitated through the enrolment survey responses and user engagement and usage metrics. These data will be collected by our collaborator, MEMOTEXT, and shared with the research team for further data cleaning and analysis. Moreover, following each iterative cycle, one-on-one interviews will be conducted with users of the SaskWell service. As mentioned, both quantitative and qualitative data will be collected to support and/or evaluate each study objective in this iterative process. A thematic analysis will be employed to summarise all qualitative study data guided by Braun and Clarke's detailed six-step process to support rigour and trustworthiness in this analysis. NVivo V.11 software will be used to conduct the thematic analysis. The research team will engage in a visual representation of the themes that are identified and seek to develop personas in the analysis and translation of study findings. A persona is an archetypal representation of user data created through an amalgamation of themes and requirements common to a group or population. ${ }^{36}$ Quantitative data will be drawn from surveying including robust demographic enrollment data. In addition to this, we will be collecting user engagement and usage metric data. Such data will include analysing the frequency of user responses to text messages, the user click rates and the duration of time in which users are engaged in the service (ie, number of users who complete the service vs number of users who drop-out of the service). In each study phase, these data will be compiled and prepared for analysis by the research team members. Furthermore, SPSS will be used for descriptive statistics such as frequencies, means and percentages to examine available reported usage data (per user), demographic variables and quantitative survey responses. Provincial population representation will drive sample size consideration in early study phases. To integrate the qualitative and quantitative findings together, the research team will employ an embedding strategy. ${ }^{37}$ An embedding strategy is often used within health intervention studies to further explore and explain the outcomes of the intervention and results. ${ }^{37} 38$ As such, by mixing the results of the quantitative and qualitative research methods, our qualitative findings can provide added breadth and depth to the quantitative data collected. ${ }^{39}$

\section{Patient and public involvement}

A patient partner (TM) has been engaged in the development of the grant application and conception of the study design and takes part in research team meetings. Moreover, TM developed and currently chairs the Saskatchewan resident advisory committee. This committee has provided support with regards to the design of the service, as well as recruitment initiatives and feedback regarding the user experience in the service. This group will also be engaged in knowledge translation and dissemination activities.

\section{ETHICS AND DISSEMINATION}

This study obtained ethical approval from the University of Saskatchewan Research Ethics Board (REB 2281) on 30 October 2020. Participant consent is indicated through the completion and submission of the enrolment survey. All information that is collected, used and disclosed is in accordance Canadian laws and follows the Personal Information and Protection and Electronic Documents Act and Health Insurance Portability and Accountability Act. Participation is entirely voluntary, and participants are able to opt out of the service at any time by texting 'STOP' to SKWELL (759355).

The findings from this study will be communicated through a knowledge dissemination plan consisting of both formal academic activities, as well as mainstream communication with the public. In addition, an integrated knowledge translation approach is included in the study through the engagement of a Saskatchewan resident advisory committee. Planned academic activities to support dissemination include presentations at academic conferences (eg, mental health and digital health related), publications in relevant academic journals and webinar presentations through the investigator's institutions, for example, grand rounds. Mainstream communication with the public includes the use of social media (eg, Twitter, Instagram and Facebook), radio and TV interviews and research snapshots included in community organisation newsletters.

\section{DISCUSSION}

Current mental health system infrastructure was not developed to support people at the scale being experienced as a result of the COVID-19 pandemic, or in a proactive way to support population health. The Mental Health Commission of Canada has identified that the increased use of technology is essential to address this gap between need and services. ${ }^{40}$ This project explores the use of a customised two-way texting service to inform and engage Canadians with existing digital mental health tools and programmes. The use of codesign throughout the design and delivery of the service creates opportunity to identify and address key issues known to challenge digital health success. The most significant of these being the identification of potential barriers in promoting and supporting access to these existing and emerging digital mental health resources. Other aspects of the study design are meant to further understanding of the benefit and/or usefulness of the online tools themselves by having users of the SaskWell service identify the resources used most often and what, if any, changes they noted in their mental health status. In order to address population mental 
health needs, we have to increase awareness and use of digital mental health services.

The effectiveness of text messaging as a means to extend the potential reach of digital interventions requires additional examination. While it is anticipated that this lower tech approach may bridge divides that separate some vulnerable or disadvantaged groups from essential mental health supports for example, additional research is needed to demonstrate this. Building on an existing knowledge synthesis project identifying key digital resources for improved mental health across the country, this research will establish low-tech connections as a means to assess need and better match access to services for those who need it most as the challenges of the global pandemic continue.

Acknowledgements The authors would like to thank the contributions of the Saskatchewan Resident Advisory Committee and MEMOTEXT.

Contributors The study concept was co-led and developed by TR and GS. PJ contributed to the technology design, enrolment considerations and engagement methods. TM, CC and IK supported the development of the advisory committee of Saskatchewan residents. TR, GS, CC and IK supported the analytic approach developed. TR, GS, CC, and IK contributed equally to drafting and editing the protocol manuscript. All authors approved the final version of the manuscript.

Funding This study is funded by the Canadian Institutes of Health Research, Operating Grant: COVID-19 Mental Health \& Substance Use Service Needs and Delivery and the Saskatchewan Health Research Foundation. The grant numbers are not applicable.

Competing interests None declared.

Patient consent for publication Not required.

Provenance and peer review Not commissioned; externally peer reviewed.

Supplemental material This content has been supplied by the author(s). It has not been vetted by BMJ Publishing Group Limited (BMJ) and may not have been peer-reviewed. Any opinions or recommendations discussed are solely those of the author(s) and are not endorsed by BMJ. BMJ disclaims all liability and responsibility arising from any reliance placed on the content. Where the content includes any translated material, BMJ does not warrant the accuracy and reliability of the translations (including but not limited to local regulations, clinical guidelines, terminology, drug names and drug dosages), and is not responsible for any error and/or omissions arising from translation and adaptation or otherwise.

Open access This is an open access article distributed in accordance with the Creative Commons Attribution Non Commercial (CC BY-NC 4.0) license, which permits others to distribute, remix, adapt, build upon this work non-commercially, and license their derivative works on different terms, provided the original work is properly cited, appropriate credit is given, any changes made indicated, and the use is non-commercial. See: http://creativecommons.org/licenses/by-nc/4.0/.

\section{ORCID iD}

Gillian Strudwick http://orcid.org/0000-0002-1080-7372

\section{REFERENCES}

1 Daly Z, Slemon A, Richardson CG, et al. Associations between periods of COVID-19 quarantine and mental health in Canada. Psychiatry Res 2021;295:113631.

2 Figueroa CA, Aguilera A. The need for a mental health technology revolution in the COVID-19 pandemic. Front Psychiatry 2020;11:523.

3 Canadian Mental Health Association. COVID-19 and mental health: heading off an echo pandemic, 2021.

4 Centre for Addiction and Mental Health. Mental health in Canada: COVID-19 and beyond, 2020. Available: https://www.camh.ca/-/ media/files/pdfs-public-policy-submissions/covid-and-mh-policypaper-pdf.pdf [Accessed 10 Mar 2021]

5 Canadian Mental Health Association. COVID-19 effects on the mental health of vulnerable populations: Wave 1, 2020. Available: https://cmha.ca/brochure/covid-19-effects-on-the-mental-health-ofvulnerable-populations-wave-1/ [Accessed 10 Mar 2021].

6 Canadian Mental Health Association. Mental health impacts of COVID-19: Wave 2, 2020. Available: https://cmha.ca/wp-content/ uploads/2020/12/CMHA-UBC-wave-2-Summary-of-Findings-FINALEN.pdf [Accessed 10 Mar 2021].

7 Moreno C, Wykes T, Galderisi S, et al. How mental health care should change as a consequence of the COVID-19 pandemic. Lancet Psychiatry 2020;7:813-24.

8 Robillard R, Daros AR, Phillips JL, et al. Emerging new psychiatric symptoms and the worsening of pre-existing mental disorders during the COVID-19 pandemic: a Canadian multisite study: Nouveaux symptômes psychiatriques émergents et détérioration des troubles mentaux préexistants durant la pandémie de la COVID-19: une étude canadienne multisite. Can J Psychiatry 2021;66:815-26.

9 Strudwick G, Sockalingam S, Kassam I, et al. Digital interventions to support population mental health in Canada during the COVID-19 pandemic: rapid review. JMIR Ment Health 2021;8:e26550.

10 Martinez-Martin N, Dasgupta I, Carter A, et al. Ethics of digital mental health during COVID-19: crisis and opportunities. JMIR Ment Health 2020;7:e23776

11 Canadian Wireless Telecommunications Association. Facts and figures, 2020. Available: https://www.cwta.ca/facts-figures/

12 Marko-Holguin M, Cordel SL, Van Voorhees BW, et al. A two-way interactive text messaging application for low-income patients with chronic medical conditions: Design-thinking development approach. JMIR Mhealth Uhealth 2019;7:e11833.

13 Vodopivec-Jamsek V, de Jongh T, Gurol-Urganci I, et al. Mobile phone messaging for preventive health care. Cochrane Database Syst Rev 2012;12:CD007457.

14 Rathbone AL, Prescott J. The use of mobile apps and SMS messaging as physical and mental health interventions: systematic review. J Med Internet Res 2017;19:e295.

15 Brar Prayaga R, Jeong EW, Feger E, et al. Improving refill adherence in medicare patients with tailored and interactive mobile text messaging: pilot study. JMIR Mhealth Uhealth 2018;6:e30.

16 Munro S, Hui A, Salmons V, et al. SmartMom Text Messaging for Prenatal Education: A Qualitative Focus Group Study to Explore Canadian Women's Perceptions. JMIR Public Health Surveill 2017;3:e7.

17 Hall AK, Cole-Lewis H, Bernhardt JM. Mobile text messaging for health: a systematic review of reviews. Annu Rev Public Health 2015;36:393-415.

18 Chang T, Gossa W, Sharp A, et al. Text messaging as a communitybased survey tool: a pilot study. BMC Public Health 2014;14:936.

19 Manganello J, Gerstner G, Pergolino K, et al. The relationship of health literacy with use of digital technology for health information: implications for public health practice. J Public Health Manag Pract 2017;23:380-7.

20 Estacio EV, Whittle R, Protheroe J. The digital divide: examining socio-demographic factors associated with health literacy, access and use of Internet to seek health information. $J$ Health Psychol 2019;24:1668-75.

21 World Health Organization. Unleashing information technology to defeat COVID-19, 2020. Available: https://www.who.int/news/ item/20-04-2020-itu-who-joint-statement-unleashing-informationtechnology-to-defeat-covid-19 [Accessed 11 Mar 2021].

22 Simon K. Digital 2020: global digital overview., 2020. Available: https://datareportal.com/reports/digital-2020-global-digital-overview [Accessed 11 Mar 2021].

23 World Health Organization. Mental health and psychosocial considerations during the COVID-19 outbreak, 2020. Available: https://www.who.int/docs/default-source/coronaviruse/mentalhealth-considerations.pdf [Accessed 11 Mar 2021].

24 Lester R, Park JJ, Bolten LM, et al. Mobile phone short message service for adherence support and care of patients with tuberculosis infection: evidence and opportunity. J Clin Tuberc Other Mycobact Dis 2019;16:100108.

25 Hyun C, McMenamin J, Ko O, et al. Efficacy of a mobile texting app (Heptalk) in encouraging patient participation in viral hepatitis B care: development and cohort study. JMIR Mhealth Uhealth 2020;8:e15098

26 Kruse G, Park ER, Shahid NN, et al. Combining real-time ratings with qualitative interviews to develop a smoking cessation text messaging program for primary care patients. JMIR Mhealth Uhealth 2019;7:e11498.

27 Brewer LC, Fortuna KL, Jones C, et al. Back to the future: achieving health equity through health informatics and digital health. JMIR Mhealth Uhealth 2020;8:e14512. 
28 Clarke PN, Watson J, Brewer BB. From theory to practice: caring science according to watson and brewer. Nurs Sci $Q$ 2009;22:339-45.

29 Glasgow RE, Harden SM, Gaglio B, et al. RE-AIM planning and evaluation framework: adapting to new science and practice with a 20-year review. Front Public Health 2019;7:64.

30 Yoshida Y, Patil SJ, Brownson RC, et al. Using the RE-AIM framework to evaluate internal and external validity of mobile phonebased interventions in diabetes self-management education and support. J Am Med Inform Assoc 2020;27:946-56.

31 Harden SM, Smith ML, Ory MG, et al. RE-AIM in clinical, community, and corporate settings: perspectives, strategies, and recommendations to enhance public health impact. Front Public Health 2018;6:71.

32 Creswell JW, Clark VLP. Designing and conducting mixed methods research. SAGE Publications, 2017. https://books.google.ca/books? id=eTwmDwAAQBAJ

33 Braun V, Clarke V. Using thematic analysis in psychology using thematic analysis in psychology. Qual Res Psychol 2006;3:77-101.

34 Keyes CLM. The mental health continuum: from languishing to flourishing in life. J Health Soc Behav 2002;43:207-22.

35 Centre for Addiction and Mental Health. Digital COVID-19 and mental health resource list, 2020. Available: http://www.camh.ca/en/health- info/mental-health-and-covid-19/information-for-professionals/covid19-database-page [Accessed 11 Mar 2021].

36 Ferreira B, Santos G, Conte T. Identifying possible requirements using personas: A qualitative study. In: ICEIS 2017 - Proceedings of the 19th International Conference on Enterprise Information Systems, 2017.

37 Zhang W, Creswell J. The use of "mixing" procedure of mixed methods in health services research. Med Care 2013;51:10.1097/ MLR.0b013e31824642fd:e51-7.

38 Fetters MD, Curry LA, Creswell JW. Achieving integration in mixed methods designs-principles and practices. Health Serv Res 2013;48:2134-56.

39 Johnson RB, Onwuegbuzie AJ, Turner LA. Toward a definition of mixed methods research. J Mix Methods Res 2007;1:10.1177/1558689806298224:112-33.

40 Mental Health Commission of Canada. E-mental health in Canada: transforming the mental health system using technology, 2014. Available: http://www.mentalhealthcommission.ca/English/system/ files/private/document/MHCC_E-Mental_Health-Briefing_Document_ ENG.pdf 